\title{
Dynamics and stability of Bose-Einstein solitons in tilted optical lattices
}

\author{
E. Díaz, ${ }^{1}$ C. Gaul, ${ }^{2}$ R. P. A. Lima, ${ }^{1,3}$ F. Domínguez-Adame, ${ }^{1}$ and C. A. Müller ${ }^{2,4}$ \\ ${ }^{1}$ Grupo Interdisciplinar de Sistemas Complejos, Departamento de Física de Materiales, Universidad Complutense, E-28040 Madrid, Spain \\ ${ }^{2}$ Physikalisches Institut, Universität Bayreuth, D-95440 Bayreuth, Germany \\ ${ }^{3}$ Instituto de Física, Universidade Federal de Alagoas, 57072-970 Maceió-AL, Brazil \\ ${ }^{4}$ Centre for Quantum Technologies, National University of Singapore, Singapore 117543, Singapore
}

(Received 30 November 2009; published 24 May 2010)

\begin{abstract}
Bloch oscillations of Bose-Einstein condensates realize sensitive matter-wave interferometers. We investigate the dynamics and stability of bright-soliton wave packets in one-dimensional tilted optical lattices with a modulated mean-field interaction $g(t)$. By means of a time-reversal argument, we prove the stability of Bloch oscillations of breathing solitons that would be quasistatically unstable. Floquet theory shows that these breathing solitons can be more stable against certain experimental perturbations than rigid solitons or even noninteracting wave packets.
\end{abstract}

DOI: 10.1103/PhysRevA.81.051607

PACS number(s): 03.75.Lm, 37.10.Jk, 52.35.Mw

Matter-wave interferometers are established as standard tools for precision measurements of small forces. The tiny wavelength of ultracold atoms is an asset, and using BoseEinstein condensates (BECs) can greatly enhance signal-tonoise ratios [1,2]. In this context, Bloch oscillations (BOs) of wave packets in tilted periodic potentials have been proven very useful [3-6]. Much after their early prediction by Zener $[7,8]$, BOs were observed with electrons in semiconductor superlattices $[9,10]$, with cold atoms in optical lattices [11,12], and with photons in waveguide arrays $[13,14]$. BOs are very sensitive to dephasing since they rely on coherent Bragg scattering of $k$-vectors from one boundary of the Brillouin zone to the other. The slightest lattice imperfection or interaction causes random scattering of different $k$-components of a wave packet, thus broadening its momentum distribution and destroying coherent oscillations in real space.

Long-living BOs of up to $10^{4}$ cycles with period $T_{\mathrm{B}}$ [15] were achieved in a BEC experiment by tuning the scattering length between Cs atoms to zero with the help of a suitable Feshbach resonance [16,17]. At finite interaction, it appears recommendable to use stable localized wave packets, namely soliton solutions to the nonlinear Schrödinger equation, in order to minimize the detrimental effects of interaction. Bright solitons arise from a dispersion that counteracts the effect of the nonlinearity. As a rule, a soliton can only be stable if the effective mass and interaction parameter have opposite signs. In the free-space case of positive mass, bright solitons are realized with atoms that attract each other [18-20]. In a lattice, the mass becomes negative close to the edge of the Brillouin zone, and there solitons can be prepared with repulsive interaction [21]. During BO cycles, the mass $m(t)$ changes its sign periodically. Thus, the interaction parameter $g(t)$ has to change accordingly in order to respect the criterion of opposite signs; both spatial and temporal nonlinearity management schemes to this effect have been proposed within the framework of the Gross-Pitaevskii equation [22,23].

Independently, we have investigated the stability of BOs with an interaction parameter $g(t)$ that is modulated harmonically in time, and we have found a whole family of cases that yield stable BOs of breathing wave packets [24]. Conversely, many other instances of $g(t)$ result in rapid destruction of BOs. Remarkably, the stability criterion developed in Ref. [24] is obviously incompatible with the simple sign rule mentioned above. For instance, the Bloch-periodic modulations $g(t)=$ $\pm g_{0} \cos \left(2 \pi t / T_{\mathrm{B}}\right)$ with opposite signs lead both to stable BOs for the same mass $m(t)$. This prompts a question that is as fundamentally interesting as it is important for practical applications: Is solitonic stability helpful to sustain BOs in general?

In this work we show that the stability of BOs is not conditioned on wave-packet rigidity. We first introduce a bounded time in the equation of motion that predicts perfectly stable breathing solutions, in full agreement with previous results [24]. But even under these premises, rigid solitons could be expected to be more robust against experimental imperfections. We study in detail the relevant case of $\mathrm{BO}$ decay caused by magnetic fields that oscillate off phase. Contrary to expectations, we find that the breathing wave packet is more stable than the rigid soliton. For the experimentally realistic parameters chosen, the rigid soliton is very close to being noninteracting. Thus, a harmonic modulation of finite interaction turns out to effectively stabilize BOs.

In the mean-field regime, the BEC is described by the complex order parameter $\Psi(x, t)$ in a one-dimensional optical lattice potential $V(x)=V_{0} \cos ^{2}(\pi x / b)$ with spacing $b$. If the lattice is sufficiently deep, one may use a tight-binding approximation, where the condensate is represented by a single complex number $\Psi_{n}(t)$ at each lattice site [25]. Our starting point is thus the nonlinear equation of motion

$$
i \hbar \dot{\Psi}_{n}=-J\left(\Psi_{n+1}+\Psi_{n-1}\right)+F b n \Psi_{n}+g(t)\left|\Psi_{n}\right|^{2} \Psi_{n}
$$

for the order parameter with normalization $\sum_{i}\left|\Psi_{i}\right|^{2}=1$. Nearest-neighbor sites are coupled by the tunneling element $J / E \approx 4\left(V_{0} / E\right)^{3 / 4} \exp \left(-2 \sqrt{V_{0} / E}\right) / \sqrt{\pi}$, where $E=$ $\hbar^{2} \pi^{2} / 2 m b^{2}$ is the lattice recoil energy [26]. The interaction parameter $g=\sqrt{2 \omega_{x} \omega_{y} \omega_{z} \hbar m / \pi} N a / J$ is derived from the scattering length $a$, the atom number $N$, and the local ground state determined by the lattice frequencies $\omega_{i}$ [25]. The dispersion relation of this single-band model reads $\epsilon(p)=-2 J \cos (p b / \hbar)$. Its curvature or inverse mass $m^{-1}=$ $2 J b^{2} \cos (p b / \hbar) / \hbar^{2}$ determines the wave-packet dynamics under the influence of a constant force $F$ that stems from a uniform acceleration of the BEC by, for example, gravity. At 
fixed lattice geometry, the interaction parameter $g(t)$ can be controlled by external magnetic fields using suitable Feshbach resonances.

In the following, we use $J$ and $b$ as units of energy and length, respectively, and set $\hbar=1$. We tackle Eq. (1) by separating the rapidly varying Bloch phase $p(t) n$ from a smooth envelope $A(z, t)$ that moves with the center of mass $x(t)$ : $\Psi_{n}(t)=e^{i p(t) n} A(n-x(t), t) e^{i \phi(t)}$. With $p(t)=-F t, x(t)=$ $x_{0}+2 \cos (F t) / F$, and $\phi=\phi_{0}+2 \sin (F t) / F$, the envelope is found to obey the equation

$$
i \partial_{t} A=-\frac{1}{2 m(t)} \partial_{z}^{2} A+g(t)|A|^{2} A,
$$

neglecting higher spatial derivatives of $A$. Note that choosing an immobile wave packet with $p(0)=0$ as the initial condition fixes the phase for subsequent BOs. The inverse mass $m(t)^{-1}=$ $2 \cos (F t)$ oscillates rapidly, but if the interaction is tuned such that $m(t) g(t)<0$ varies slowly enough, Eq. (2) admits a soliton solution

$$
A(z, t)=\frac{1}{\sqrt{2 \xi}} \frac{1}{\cosh (z / \xi)} e^{-i \omega t},
$$

whose quasistatic width is $\xi=-2 /[g(t) m(t)]>0$. To be stable, the soliton must be able to follow this width adiabatically. Otherwise, its breathing mode will be driven, and other excitations may be created. Therefore, the least disruptive way of accelerating a soliton of width $\xi_{0}$ is to impose a perfectly rigid envelope by choosing $g_{\mathrm{r}}(t)=-2 m(t)^{-1} / \xi_{0}=$ $g_{\mathrm{r}} \cos (F t)$ with $g_{\mathrm{r}}=-4 / \xi_{0}<0$. More extensive studies based on this idea have been put forward in Refs. [22,23].

Let us contrast this reasoning with an analytical timereversal argument that we developed after studying the stability of BOs under harmonic modulations of $g$ [24]. Quite generally, a rigid wave packet is by no means necessary for persistent BOs. Already for the case $g(t)=0$, breathing is the rule: In the first quarter of the Bloch cycle, the mass is positive and the wave packet spreads. When the mass changes sign, the time evolution is reversed and the wave packet recovers its original shape at the edge of the Brillouin zone. Thus, the wave packet shows periodic breathing on top of the BO, independently of its initial shape. Also in the interacting case, one can find nontrivial functions $g(t)$ in Eq. (2) compatible with this time reversal. Consider the class of periodic functions

$$
g(t)=\cos (F t) P(\sin (F t) / F),
$$

where a factor $\cos (F t)$ can be separated from a polynomial $P(\eta)$ in the bounded time variable

$$
\eta(t)=\frac{1}{2} \int_{0}^{t} m(s)^{-1} d s=\frac{\sin (F t)}{F} .
$$

Because $\partial_{t} \eta(t)=[2 m(t)]^{-1}$, the explicit time dependence of the mass factorizes from all terms in equation of motion (2) for $A(z, t)=\tilde{A}(z, \eta(t))$ :

$$
i \partial_{\eta} \tilde{A}(z, \eta)=-\partial_{z}^{2} \tilde{A}(z, \eta)+P(\eta)|\tilde{A}|^{2} \tilde{A}(z, \eta) .
$$

The ensuing dynamics for $\tilde{A}(z, \eta)$ as function of $\eta$ may be quite complicated. However, as $\eta(t)$ itself is a periodic function of time, the solution $A(z, t)$ must also be periodic: Any dynamics taking place in the first quarter of the Bloch period, while $\eta$ runs

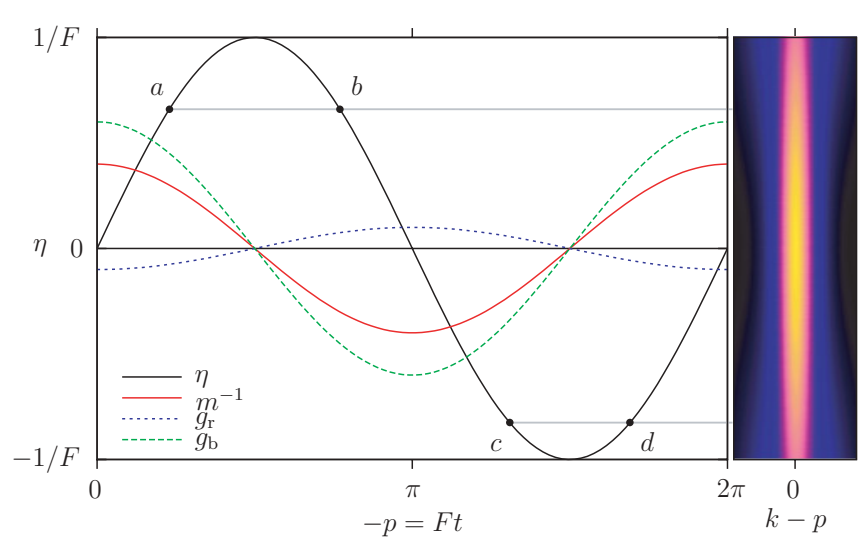

FIG. 1. (Color online) Time evolution scheme of stable BOs. Left: The inverse mass $m^{-1}$, the interaction parameter of the rigid soliton $g_{\mathrm{r}}$, as well as that of a breathing soliton $g_{\mathrm{b}}$, are shown together with the bounded time $\eta=\sin (F t) / F$ as function of time or momentum $-p=$ $F t$. Right: The $k$-space density [obtained by numerical integration of Eq. (1)] of a breathing wave packet is a function of $\eta$ and thus strictly periodic in $t$; the points in time $a$ and $b$ as well as $c$ and $d$ show the same distribution, respectively.

from 0 to $1 / F$, is exactly reversed in the next quarter, when $\eta$ runs back. Figure 1 illustrates this argument by showing the time dependence of several key quantities over one Bloch cycle, as well as a $k$-space density plot with clearly visible breathing dynamics. ${ }^{1}$

We stress that Eq. (4) includes both cases, $g(t)=$ $\pm g_{0} \cos (F t)$. Although the $+\cos$ case does not fulfill the soliton stability criterion $m(t) g(t)<0$, the preceding timereversal argument ensures that both cases lead to undamped Bloch oscillations - at least within the approximations underlying equation of motion (2). As shown in the right-hand panel of Fig. 1, this prediction is confirmed by numerical integration of the tight-binding model (1) with a standard fourth-order Runge-Kutta method.

Which of these stable solutions are the most robust under variations of experimental control parameters? Indeed, even cold-atom experiments suffer from slight imperfections such as residual uncertainties in the magnetic field controlling the interaction term $g(t)$. For instance, in the Innsbruck experiment [15], the magnetic field is controlled up to $1 \mathrm{mG}$. The slope of $61 a_{0} / \mathrm{G}$ at the zero of the Feshbach resonance turns this into an uncertainty $\Delta a=0.06 a_{0}$ in the scattering length, which is converted to the uncertainty of the dimensionless tight-binding interaction $\Delta g \approx 0.4$. Note that this uncertainty $\Delta g$ is larger than the interaction $g_{\mathrm{r}}=-4 / \xi_{0}$ needed to create a rigid soliton of only moderate width $\xi_{0} \gtrsim 10$. From this point of view, realizing a wide rigid soliton is practically equivalent to switching the interaction off altogether.

We study numerically the effect of perturbations of $g(t)$ by numerical integration of Eq. (1). Rather than the strong force $F \approx 34$ in a vertical lattice [15], we choose a smaller force

\footnotetext{
${ }^{1}$ Equation (4) covers all stable cases that are Bloch periodic; that is, $v_{2}=1$ in Eq. (8) of [24]. The cases $v_{2} \neq 1$ are also covered by generalizing the bounded-time argument [27].
} 


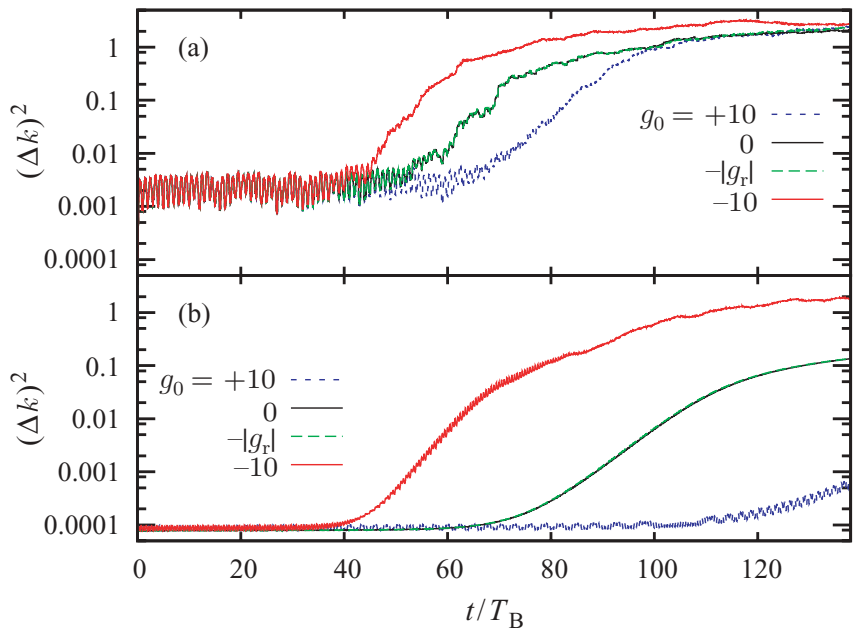

FIG. 2. (Color online) Momentum width $(\Delta k)^{2}$ of a wave packet, Eq. (3), with initial spatial width $\xi=66.16$ (plus a small seed noise of $10^{-3}$ mimicking experimental inhomogeneities), Bloch-oscillating in a tilted lattice with $F=2 \pi / T_{\mathrm{B}}=0.15$. The interaction parameter is modulated as $g(t)=g_{0} \cos (F t)+v(t)$. (a) Random perturbation with frequencies $\Omega_{n}=n F / N$ below and above the Bloch frequency $v(t)=\sum_{n=1}^{N^{2}} \operatorname{Re}\left[a_{n} \exp \left(i \Omega_{n} t\right)\right], N=5$. The $a_{n}$ are complex random numbers with $\overline{a_{n}}=0, \overline{\left|a_{n}\right|^{2}}=0.5$. (b) Sine perturbation $v(t)=$ $0.5 \sin (F t)$. In all cases the lifetime increases with the perturbation amplitude $g_{0}$, from the antibreathing wave packet $g_{0}=-10$, over the rigid soliton $g_{0}=g_{\mathrm{r}}=-0.06$ and the linear wave packet $g_{0}=0$, to the breathing soliton $g_{0}=+10$.

$F=0.15$, corresponding to a slighter tilt. This results in a longer Bloch period and a higher sensitivity to dephasing. The broadening of the momentum distribution is directly measurable from the experimental time-of-flight images and it signals the decay of the wave packet and destruction of BOs in real space. In Fig. 2, the $k$-space variance $(\Delta k)^{2}$ is shown for $g(t)=$ $g_{0} \cos (F t)+v(t)$ with two different types of perturbations $v(t)$ and different modulation strengths $g_{0}$. In all cases, the momentum distribution starts to broaden at a certain time. At a given perturbation, the rigid soliton and the linear wave packet show greater resilience than the strongly antibreathing wave packet, but surprisingly the breathing wave packet survives even longer. Obviously, the $+\cos$ modulation stabilizes the Bloch oscillations against uncontrolled variations of the interaction parameter $g$.

Comparing a random superposition of different frequencies [Fig. 2(a)] to a Bloch-periodic off-phase perturbation proportional to $\sin (F t)$ [Fig. 2(b)], we trace back the differences in lifetime to different sensitivities to the sine perturbation. In the following, we thus consider $g(t)=g_{0} \cos (F t)+g_{1} \sin (F t)$ with an off-phase perturbation with amplitude $g_{1}$ of order $\Delta g$. In order to understand quantitatively why the + cos-modulated wave packet can be more robust than the rigid soliton, we perform a homogeneous stability analysis. The sudden growth of the momentum variance suggests an instability due to the creation of small fluctuations. If these perturbations occur on a length scale much shorter than the size of the wave packet, the wave packet can be taken to be locally homogeneous: $|A(z, t)|^{2} \approx n_{0}=1 / 2 \xi$. The real and imaginary parts of the

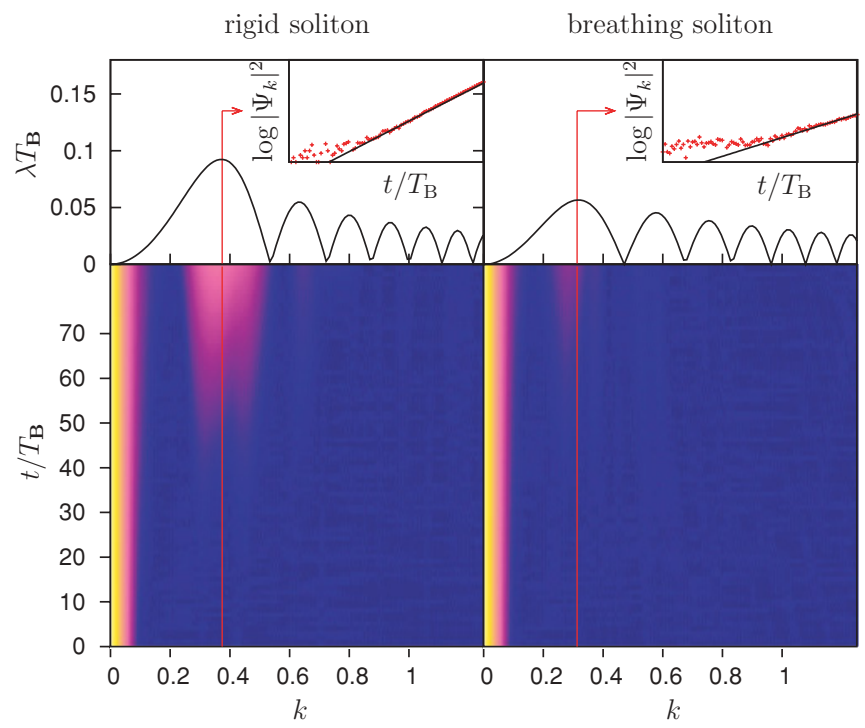

FIG. 3. (Color online) Decay of a (left) rigid and a (right) breathing soliton under the harmonic perturbation of Fig. 2(b). Upper panel, linear stability growth rate, Eq. (8); lower panel, stroboscopic plot of the $k$-space density on a logarithmic color scale; inset, the location and growth of the most unstable mode agree with the analytical prediction.

small fluctuations in the linear order, $s$ and $d$, respectively, obey equations of motion that decouple in Fourier modes [24]:

$$
\begin{gathered}
\dot{d}_{k}=-\left[k^{2} \cos (F t)+2 n_{0} g(t)\right] s_{k}, \\
\dot{s}_{k}=k^{2} \cos (F t) d_{k} .
\end{gathered}
$$

Thanks to their linearity and time periodicity, these equations allow us to apply Floquet theory [28]. Integrating Eq. (7) over a single period allows calculation of the Lyapunov exponent $\lambda_{k}$ that characterizes the exponential growth of mode $k$. For the present case, $g(t)=g_{0} \cos (F t)+g_{1} \sin (F t)$, Eq. (7) can be solved analytically for $g_{1}=0$, using a Bogoliubov transformation $\gamma_{k}=\sqrt{\omega_{k} / k^{2}} s_{k}+i \sqrt{k^{2} / \omega_{k}} d_{k}$, with $\omega_{k}=\sqrt{k^{2}\left(k^{2}+2 n_{0} g_{0}\right)}$. Perturbation theory to first order in $g_{1}$ then predicts the growth of mode $\gamma_{k}$ with

$$
\lambda_{k}=k^{2}\left|\frac{g_{1} n_{0}}{\omega_{k}} J_{1}\left(2 \omega_{k} / F\right)\right|,
$$

where $J_{1}$ is the Bessel function of the first kind. For the two cases of primary interest-the rigid soliton and the breathing soliton-the top of Fig. 3 shows this Lyapunov exponent, which is indistinguishable from the value obtained by numerical solution of Eq. (7), as function of $k$. Mainly the prefactor $k^{2} /\left|\omega_{k}\right|=\left|1+2 g_{0} n_{0} / k^{2}\right|^{-1}$ makes the Lyapunov exponents of the breathing wave packet $\left(g_{0}=10\right)$ smaller than those of the rigid soliton $\left(g_{0}=g_{\mathrm{r}}=-0.06\right){ }^{2}$

The Lyapunov exponent (8) provides a rather faithful portrait of the $k$-space evolution as obtained by the numerics, plotted in the lower panels of Fig. 3. Notably, excitations

\footnotetext{
${ }^{2}$ For $g_{0}<0$, very small $k$-values such that $k^{2}<2 n_{0}\left|g_{0}\right|$ have imaginary Bogoliubov frequencies $\omega_{k}$, but Eq. (8) allows for a smooth analytic continuation (cf. Fig. 3).
} 
grow exclusively in the intervals with the largest Lyapunov exponents, which are found at $k$-values such that $k^{2} \gg n_{0} g_{0}$. Thus, the most unstable mode (indicated by the vertical line) is found close to $k_{*}$ defined by $J_{1}^{\prime}\left(2 k_{*}^{2} / F\right)=0$, which gives $k_{*} \approx 0.96 \sqrt{F} \approx 0.37$. The predicted growth rate agrees very well with the numerical data (inset in the top panel of Fig. 3). These predictions also remain valid for smaller $g_{0}$. In the limit $g_{0} \rightarrow 0$, the differences between different wave packets disappear, and we recover the case of a pure $\sin (F t)$ modulation that was analyzed in Ref. [24] and for which Eq. (8) now provides an analytical expression.

In essence, this linear-stability analysis applies whenever the excitations are well decoupled in $k$-space from the original wave packet (the central peak around $k=0$ visible in Fig. 3). Note that other types of perturbations, for example, a constant offset $g_{2}$ as in $g(t)=g_{0} \cos (F t)+g_{2}$ [29], can cause a more homogeneous broadening of the $k$-space distribution. Its effect is better captured by an ansatz in terms of collective variables (cf. [24]). A detailed analysis is beyond the scope of the present work and will be addressed in a forthcoming publication.

In conclusion, we have connected the physics of solitons in lattices with a stability analysis of BOs under harmonic variations of the interaction. The stability of BOs does not rely on a stable soliton configuration. Instead, the wave packet may start to fall apart, but it comes back by virtue of periodic time reversal. In the presence of instability-inducing perturbations, a modulation of the interaction can make the wave packet more robust. We explain this behavior quantitatively via linear stability analysis within Floquet theory. Finally, let us stress that these results play an important role in the design of accurate and reliable matter-wave interferometers based on BOs.

Travel between Bayreuth and Madrid was supported by the joint program Acciones Integradas of Deutscher Akademischer Auslandsdienst (DAAD) and Ministerio de Educación y Ciencia (MEC). C.G. and C.A.M acknowledge financial support from Deutsche Forschungsgemeinschaft (DFG), and thank H.-C. Nägerl and his group for hospitality and helpful discussions. Work at Madrid was supported by MEC (Project MOSAICO). Work at Maceió was partially supported by the Brazilian research agencies Conselho Nacional de Desenvolvimento Científico e Tecnológico $(\mathrm{CNPq})$ and Coordenação de Aperfeiçoamento de Pessoal de Nível Superior (CAPES).
[1] P. Bouyer and M. A. Kasevich, Phys. Rev. A 56, R1083 (1997).

[2] A. K. Tuchman and M. A. Kasevich, Phys. Rev. Lett. 103, 130403 (2009).

[3] P. Cladé, S. Guellati-Khélifa, F. Nez, and F. Biraben, Phys. Rev. Lett. 102, 240402 (2009).

[4] H. Müller, S. W. Chiow, S. Herrmann, and S. Chu, Phys. Rev. Lett. 102, 240403 (2009).

[5] E. Haller et al., Science 325, 1224 (2009).

[6] M. Gustavsson et al., e-print arXiv:0812.4836 (2008).

[7] F. Bloch, Z. Phys. 52, 555 (1929).

[8] C. Zener, Proc. R. Soc. London A 145, 523 (1934).

[9] J. Feldmann et al., Phys. Rev. B 46, 7252 (1992).

[10] K. Leo, P. H. Bolivar, F. Brüggemann, R. Schwedler, and K. Köhler, Solid State Commun. 84, 943 (1992).

[11] M. Ben Dahan, E. Peik, J. Reichel, Y. Castin, and C. Salomon, Phys. Rev. Lett. 76, 4508 (1996).

[12] B. P. Anderson and M. A. Kasevich, Science 282, 1686 (1998).

[13] T. Pertsch, P. Dannberg, W. Elflein, A. Brauer, and F. Lederer, Phys. Rev. Lett. 83, 4752 (1999).

[14] R. Morandotti, U. Peschel, J. S. Aitchison, H. S. Eisenberg, and Y. Silberberg, Phys. Rev. Lett. 83, 4756 (1999).

[15] M. Gustavsson et al., Phys. Rev. Lett. 100, 080404 (2008).
[16] E. A. Donley et al., Nature 412, 295 (2001).

[17] T. Köhler, K. Góral, and P. S. Julienne, Rev. Mod. Phys. 78, 1311 (2006).

[18] V. M. Pérez-García, H. Michinel, and H. Herrero, Phys. Rev. A 57, 3837 (1998).

[19] L. Khaykovich et al., Science 296, 1290 (2002).

[20] K. E. Strecker, G. B. Partridge, A. G. Truscott, and R. G. Hulet, Nature 417, 150 (2002).

[21] B. Eiermann et al., Phys. Rev. Lett. 92, 230401 (2004).

[22] M. Salerno, V. V. Konotop, and Y. V. Bludov, Phys. Rev. Lett. 101, 030405 (2008).

[23] Y. V. Bludov, V. V. Konotop, and M. Salerno, J. Phys. B 42, 105302 (2009).

[24] C. Gaul, R. P. A. Lima, E. Díaz, C. A. Müller, and F. DomínguezAdame, Phys. Rev. Lett. 102, 255303 (2009).

[25] A. Trombettoni and A. Smerzi, Phys. Rev. Lett. 86, 2353 (2001).

[26] O. Morsch and M. Oberthaler, Rev. Mod. Phys. 78, 179 (2006).

[27] C. Gaul, Ph.D. thesis, Universität Bayreuth, 2010, [http://opus.ub.uni-bayreuth.de/volltexte/2010/678/].

[28] N. G. Markley, Principles of Differential Equations (Wiley, Hoboken, NJ, 2004), Ch. 5.4.

[29] J. Gong, L. Morales-Molina, and P. Hänggi, Phys. Rev. Lett. 103, 133002 (2009). 